\title{
Biodiversidad antropoentomofágica de la región de Zongolica, Veracruz, México
}

\author{
Julieta Ramos-Elorduy ${ }^{1}$, Ivonne Landero-Torres ${ }^{2}$, Joaquín Murguía-González ${ }^{2}$ \& José M. Pino M. ${ }^{1}$ \\ 1 Instituto de Biología, Universidad Nacional Autónoma de México, Departamento de Zoología, México D.F., México. \\ P.O. Box. 70-153, 04510 México D.F., México. Fax: (+55) 5550-0164; relorduy@ibiologia.unam.mx \\ 2 Facultad de Ciencias Biológicas y Agropecuarias, Universidad Veracruzana Córdoba, Ver. Km 1 carretera Peñuela \\ Amatlán de los Reyes Ver.
}

Recibido 01-XI-2006. Corregido 14-V-2007. Aceptado 08-VI-2007.

\begin{abstract}
Anthropoentomophagic biodiversity of the Zongolica region, Veracruz, Mexico. During two and a half years (2003-2005) we recorded the insect species used as food at Zongolica, Veracruz State, Mexico. Interviews were made among people (200) of this municipality to know which insects they consumed. The total of registered species was 57 (Orthoptera, Hemiptera, Homoptera, Megaloptera, Coleoptera, Lepidoptera and Hymenoptera). The Orthoptera was the most frequently ingested. Twenty-four of these species were new records for edible insects of Mexico. They are eaten in immature stages or as adults, generally only roasted. Consumption is seasonal. Some species are commercialized in the "tianguis" (little town markets) and/or in the larger Zongolica market. There is a "protoculture" of three species, one cockroach (Periplaneta australasiae Fabricius) and two moths (Latebraria amphipyroides Guenée and Arsenura armida armida Cramer). In Zongolica, anthropoentomophagy is an ancestral habit. Rev. Biol. Trop. 56 (1): 303-316. Epub 2008 March 31.
\end{abstract}

Key words: edible insects, biodiversity, Zongolica, Veracruz, Mexico.

La Etnoentomología estudia las interrelaciones humanas con el mundo de los insectos. Gabdin (1973) y Hogue (1987) la definen como "todas las formas de interacción entre los insectos y el hombre, especialmente entre las sociedades humanas primitivas y no industrializadas". En ella se involucran diversas áreas de diferentes disciplinas, como la alimentación, la medicina, la historia, la antropología, la lingüística, la sociología, la taxonomía, la etología, la mística, la artesanía y el arte (literario, pictórico, escultórico, textil y cinematográfico). Recientemente se maneja el concepto de antropoentomofagía erigido por Costa-Neto (2002) para diferenciar la "entomofagía" que realizan muchos animales silvestres y domésticos e incluso las plantas insectívoras, del consumo de insectos efectuado por el ser humano. En la actualidad hemos censado 545 especies de insectos comestibles en la República Mexicana (Ramos-Elorduy 2000).

Los insectos comestibles forman parte de la alimentación de muchas comunidades rurales en el mundo (Ramos-Elorduy y Conconi 1994), proveyéndolos de una cantidad importante de nutrientes, sobre todo de proteínas (Ramos-Elorduy et al. 1984, Ramos-Elorduy 1991, 2005). Se consumen en todos los estados de desarrollo, pero principalmente en los inmaduros (Ramos-Elorduy 2000). En algunos lugares de África, que es donde se ha censado, los insectos llegan a constituir del $51 \%$ al 81 $\%$ del total de la proteína animal que consumen (Gómez et al. 1961, Bahuchet 1972, 1978, Kitsa 1989). Existe una secuenciación en su consumo y las especies ingeridas varían de una estación a otra, pero en cada una se consumen diferentes tipos de ellas (Ramos-Elorduy y 
Pino 1998, 2001a, 2002b), muchas especies se llegan a almacenar para contar con alimentos cotidianamente. Algunas especies incluso se comercializan en diferentes grados, y llegan a abarcar el mercado internacional (RamosElorduy 2005). Actualmente constituyen una delicatessen en varios países del primer mundo y de Europa y tienen una gran aceptación y demanda (Gordon 1998, Menzel and D'Aluisio 1998, Ramos-Elorduy 1998).

Dado que la región de Zongolica está parcialmente aislada, enclavada en la Sierra Madre Oriental, supusimos que por su origen Náhuatl, la antropoentomofagia era muy practicada, ya que hasta ahora este es el grupo étnico más entomófago (Ramos-Elorduy y Pino 2002a), comprobándose así que esta es un hábito ancestral (Ramos-Elorduy 1999) que persiste. Además conocer que especies de insectos consumen y la forma cómo se ingieren y también saber si se comercializan y si alguna (s), de ella (s) se cultivan.

\section{MATERIALES Y MÉTODOS}

Lugar de estudio: el municipio de Zongolica en el estado de Veracruz, se encuentra situado en la zona central montañosa,

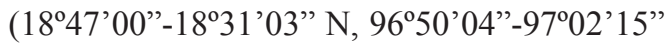
W). Su altitud es de 1200 metros. Limita con los municipios de Tequila, Omealca, Tezonapa Los Reyes, Texhuacán, Mixtla de Altamirano, Tehuipango, y al sur y suroeste con el estado de Puebla. Tiene una extensión de $347.33 \mathrm{~km}^{2}$ que representa el $0.0046 \%$ del total del estado y el $0.00017 \%$ del país. Cuenta con una población de 39562 habitantes (INEGI 2000). El grupo étnico que predomina es el náhuatl. El clima es templado-húmedo extremoso $\mathrm{Cwb}$ (García 1964) con temperatura media anual de $18.6{ }^{\circ} \mathrm{C}$ con lluvias abundantes en verano. La vegetación es de tipo selva alta perennifolia, cuenta con especies de cedro (Cedrela mexicana Roem), palo mulato (Bursera simaruba Serg.) y guarumbo (Cecropia peltata Linneo). Existe una amplia diversidad de especies animales como son: temazate (Mazama americana
Erxleben), tepezcuintle (Agouti paca Linneo), conejo (Sylvilagus sp.), tejón (Nasua narica Linneo), mapache (Procyon lotor Linneo), ardilla (Sciurus sp. y Spermophilus sp.), zorro (Urocyon cinereoargenteus Schreber) entre otros. La agricultura de la zona es de cultivos de maíz (Zea mays Linneo), café (Coffea arabiga Linneo), mango (Mangifera indica Linneo), pera (Pyrus communis Linneo), naranja (Citrus aurantium Linneo) y manzana (Malus sylvestris Mill.), la explotación forestal es principalmente de guarumbo ( $C$. peltata), caoba (Swietenia macrophyla King), palo mulato (B. simaruba), cedro (C. mexicana) y xochicuahuitl (Cordia ferruginea Roem et Schult.).

La agricultura de los pueblos nahuas se apoya en la alternancia del cultivo de maíz y frijol, que son indispensables para su economía de subsistencia, lo que continúa realizándose con el sistema de roza, tumba y quema, en donde recurren a los ritos propiciatorios, para alcanzar el favor y los dones de la naturaleza.

El uso y trabajo forestal son practicados en la mayoría de las comunidades de tierra fría. Recientemente se han introducido técnicas alternativas agroecológicas con resultados satisfactorios en el uso de plantas frutales, nopales y magueyes como tecnología de conservación de suelos, aspecto fundamental en la parte serrana, en donde las pendientes e inclinaciones sólo permiten el cultivo reducido en ladera.

Podemos decir que la ganadería de especies pequeñas, como la ovicaprina, se mantiene en todas las comunidades indígenas de la tierra fría y se practica en forma tradicional, tanto en el traspatio de la casa familiar como en terrenos de pastoreo. La fauna nativa aún es muy rica a pesar del número de especies que utilizan los indígenas.

El trabajo de prospección se realizó durante dos años y medio, a partir de enero 2003 a octubre del 2005, realizando visitas mensuales, en ocho localidades: Zongolica, La Quinta, Ixcohuapa, La Laguna, Ixtapaluka, San Juan, Tequila y Amatitla.

Entrevistas: las entrevistas aplicadas para conocer que insectos comían, fueron de tipo 
semiestructurada, por lo tanto es una investigación con enfoque eticista, que es la perspectiva académica o sea el punto de vista del investigador, pero buscando los aspectos étnicos que es el conocimiento de la gente local, desde el punto de vista etnocientífico o sea, es el punto de vista de los campesinos (Costa-Neto 2002). Las entrevistas se realizaron con lugareños (200) elegidos al azar, en los poblados mencionados, alrededor del municipio de Zongolica al igual que en éste, entrevistándolos en sus casas, siendo éstos de ambos géneros, cuyas edades oscilaron de los 18 a 37 años, efectuándolas una vez que contábamos con su anuencia para responder el cuestionario, con la finalidad de obtener la información buscada sobre las diversas especies de insectos comestibles en la zona de estudio.

Las entrevistas fueron sincrónicas (las preguntas del cuestionario se realizaban a diferentes personas en un lapso corto de tiempo) y diacrónicas (las mismas preguntas, se les formularon a las mismas personas en diferente tiempo, alrededor de un año después) y se presentan los valores medios obtenidos de éstas.

Cuestionario para conocer la manera de identificar preparar y determinar el hábito antropoentomofágico en la región de Zongolica, Veracruz, México.

1. ¿Qué animalitos come? (Chapulines, abejas, gusanos de los palos, toritos, cuetla, otros)

2. ¿Cómo los reconoce? (Tamaño, color, forma, olor, textura)

3. ¿Están en...? (Selva; Arroyo; Hierba; Árboles; Arbustos; Tierra)

4. ¿Sabe dónde encontrarlos? (Los busca o conoce el lugar)

5. Su presencia y abundancia la relaciona con... (Lluvia; Seco; Frío; Calor; Humedad)

6. ¿Cómo los recoge? (A mano, Canastos, Redes, Palas, Hachas)

7. ¿Desde cuándo los busca y come? (Desde niño, hace poco tiempo, hace algunos años)
8. ¿Quién se los enseñó a comer? (Mamá, abuelos, amigos, lo ví)

9. ¿Quién los prepara? (Mamá, yo mismo, otros, los compra)

10. ¿Cómo los comen? (Asados, fritos, en sopa, guisados, vivos)

11. ¿Sabe si tienen otros usos? (Medicina, diversión, otros)

12. ¿Tienen alguna forma de cultivarlos? ( $\mathrm{Si}$, No)

Mercadeo: posteriormente se visitaron los mercados y tianguis de las diversas localidades para ver si alguna especie se vendía y cómo (secas, preparadas listas para ingerirse, por medidas, por peso, por puños o por número etc.).

Recolecta: concomitantemente junto con algunos lugareños que nos acompañaban en los recorridos a través del área de estudio para buscar a las especies comestibles de insectos que nos habían comentado consumían, se recolectaron a éstas conforme nos indicaban dónde y cómo los obtenían, dependiendo de la estación del año en que nos encontráramos y para éste efecto se emplearon pinzas entomológicas, redes y también se capturaron manualmente. Posteriormente, nos mostraron cómo las preparaban, ingerían y cómo algunas las vendían y/o almacenaban.

Identificación: los ejemplares se depositaron en frascos con alcohol al $70 \%$ poniendo en cada uno los datos correspondientes a fecha, localidad, colector, hospedero y observaciones particulares. El material así preparado se trasladó al Departamento de Zoología del Instituto de Biología de la Universidad Nacional Autónoma de México (UNAM) para su montaje, etiquetado y determinación taxonómica mediante el empleo de claves, siendo en algunos casos ratificados con los especialistas de esos órdenes. Los ejemplares están depositados en la Colección Nacional de Insectos Comestibles de la UNAM. 


\section{RESULTADOS}

Entrevistas: los resultados de las entrevistas (Cuadro 1) arrojaron los siguientes datos: los habitantes de la Región de Zongolica conocen varios tipos de insectos que se consumen, siendo ampliamente conocidos los chapulines y la cuetla $23 \%$ y las abejas $20 \%$. En la pregunta de que otros animalitos se obtuvo un $27 \%$, observando que identifican entre otros insectos comestibles, los panales (avispas), la cuecla, las chicatanas, además de otras especies. Los

CUADRO 1

Porcentaje de percepción antropoentomofágica en el municipio de Zongolica, Veracruz, México

TABLE 1

Percentage of anthropoentomophagic perception at Zongolica, Veracruz, México

\begin{tabular}{|c|c|c|c|c|c|c|}
\hline $\begin{array}{l}\text { Insectos } \\
\text { Preguntas }\end{array}$ & $\begin{array}{l}\text { Chapulines } \\
\text { (1) }\end{array}$ & $\begin{array}{l}\text { Abejas } \\
\text { (2) }\end{array}$ & $\begin{array}{l}\text { Gusanos de } \\
\text { los palos(3) }\end{array}$ & $\begin{array}{l}\text { Toritos } \\
\text { (4) }\end{array}$ & $\begin{array}{l}\text { Cuetla } \\
\text { (5) }\end{array}$ & $\begin{array}{c}\text { Otros } \\
(6)\end{array}$ \\
\hline ¿Qué animalitos come?* & 23 & 20 & 16 & 7 & 23 & 27 \\
\hline \multirow[t]{2}{*}{ ¿Cómo los reconoce? } & Tamaño & Color & Forma & Olor & Textura & \\
\hline & 30 & 19 & 48 & 3 & 0 & \\
\hline \multirow[t]{2}{*}{ ¿Están en...? * } & Selva & Arroyo & Hierba & Árboles & Arbustos & Tierra \\
\hline & 0 & 2 & 35 & 37 & 26 & 28 \\
\hline \multirow[t]{2}{*}{ ¿Sabe donde encontrarlos? } & Los busca & $\begin{array}{l}\text { Conoce } \\
\text { el lugar }\end{array}$ & & & & \\
\hline & 22 & 78 & & & & \\
\hline \multirow{2}{*}{$\begin{array}{l}\text { Su presencia y abundancia la } \\
\text { relaciona con... }\end{array}$} & Lluvia & Seco & Frío & Calor & Humedad & \\
\hline & 45 & 27 & 26 & 28 & 0 & \\
\hline \multirow[t]{2}{*}{ ¿Como los recoge? } & A mano & Canastos & Redes & Palas & Hachas & \\
\hline & 48 & 32 & 0 & 1 & 19 & \\
\hline \multirow[t]{2}{*}{$\begin{array}{l}\text { ¿Desde cuándo los busca y } \\
\text { come? }\end{array}$} & Desde Niño & $\begin{array}{l}\text { Hace poco } \\
\text { tiempo }\end{array}$ & $\begin{array}{l}\text { Hace algunos } \\
\text { años }\end{array}$ & & & \\
\hline & 78 & 5 & 17 & & & \\
\hline \multirow[t]{2}{*}{ ¿Quién se los enseño a comer? } & Mamá & Abuelos & Amigos & Lo ví & & \\
\hline & 72 & 23 & 5 & 0 & & \\
\hline
\end{tabular}


CUADRO 1 (continuación...)

Porcentaje de percepción antropoentomofágica en el municipio de Zongolica, Veracruz, México

TABLE 1 (continued...)

Percentage of anthropoentomophagic perception at Zongolica, Veracruz, México

\begin{tabular}{|c|c|c|c|c|c|c|}
\hline $\begin{array}{l}\text { Insectos } \\
\text { Preguntas }\end{array}$ & $\begin{array}{l}\text { Chapulines } \\
\text { (1) }\end{array}$ & $\begin{array}{l}\text { Abejas } \\
(2)\end{array}$ & $\begin{array}{l}\text { Gusanos de } \\
\text { los palos(3) }\end{array}$ & $\begin{array}{l}\text { Toritos } \\
\text { (4) }\end{array}$ & $\begin{array}{l}\text { Cuetla } \\
(5)\end{array}$ & $\begin{array}{c}\text { Otros } \\
(6)\end{array}$ \\
\hline \multirow[t]{2}{*}{ ¿Quién los prepara? } & Mamá & Yo mismo & Otros & Los compra & & \\
\hline & 73 & 20 & 0 & 7 & & \\
\hline \multirow[t]{2}{*}{ ¿Cómo los comen? } & Asados & Fritos & Sopa & Guisados & Vivos & \\
\hline & 69 & 12 & 4 & 15 & 0 & \\
\hline \multirow[t]{2}{*}{ ¿Sabe si tienen otros usos? } & Medicina & Diversión & Otras & & & \\
\hline & 86 & 14 & 0 & & & \\
\hline \multirow{2}{*}{$\begin{array}{l}\text { ¿Tienen alguna forma de } \\
\text { cultivarlos? }\end{array}$} & Sí & No & & & & \\
\hline & 7 & 93 & & & & \\
\hline
\end{tabular}

+ Se presenta la media obtenida de la aplicación del mismo cuestionario dos veces.

* Dieron más de una respuesta.

1) Sphenarium spp., Melanoplus spp., Taeniopoda spp. (Orthoptera-Acrididae) 2) Apis mellifera, Partamona bilineata, (Hymenoptera-Apidae) 3) Callipogon sp., Chalcophora sp. (Coleoptera-Cerambycidae), 4) Umbonia spp. (HomopteraMembracidae), 5) Latebraria amphipyroides (Lepidoptera-Noctuidae) 6) Stilpnochlora spp. (esperanzas) (OrthopteraTettigonidae), Periplaneta spp. (cucarachas) (Orthoptera-Blattidae), Fidicinoides epigea (Cigarras) (Homoptera-Cicadidae), Corydalus sp. (manfes) (Megaloptera-Corydalidae), Phyllophaga sp., Goliathus goliathus, Geotrupes sp. (gusanos de la tierra) (Coleoptera-Melolonthidae), Pseudodirphia mexicana (Lepidoptera-Arctiidae), Helicoverpa zea (Lepidoptera-Noctuidae), Phasus triangularis (gusanos) (Lepidoptera-Hepialidae), Arsenura armida armida (cuecla) (Lepidoptera-Saturniidae), Atta spp. (chicatanas) (Hymenoptera-Formicidae), Polistes spp., Polybia spp. (avispas)(Hymenoptera-Vespidae).

reconocen principalmente por su forma $48 \% \mathrm{y}$ por su tamaño $30 \%$.

En cuanto al lugar en donde se les localiza el $37 \%$ contestó que en los árboles, el $35 \%$, en la hierba, el $26 \%$ en los arbustos y sólo el $2 \%$ en los arroyos.

La mayor parte de la gente los busca en su época de abundancia, y el $78 \%$ sabe el lugar donde encontrarlos. Su presencia la relacionan sobre todo con la época de lluvias $45 \%$ y con el calor $28 \%$. Los capturan el $48 \%$ manualmente, el $32 \%$ con ayuda de cestos o canastos
(Fig. 1), el $20 \%$ con diversos implementos agrícolas, ninguno emplea redes.

Con relación a la pregunta ¿desde cuándo los consume?, el $78 \%$ contestó que desde niño, a la de ¿quién se los enseñó a comer? el $72 \%$ respondió que su mamá y el $23 \%$ sus abuelos. Generalmente los prepara su mamá o esposa 73 $\%$, ó ellos mismos, el $20 \%$. Con respecto a la forma en que los ingieren el $69 \%$ los consume asados, ninguna especie es ingerida viva.

Además conocen el hecho de que también se emplean en la medicina tradicional $86 \%$, y 


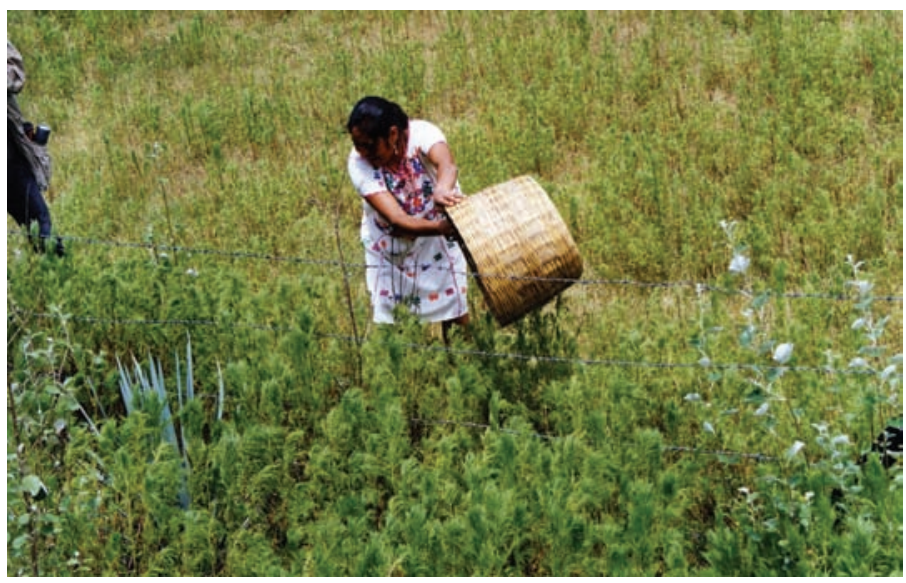

Fig. 1. Recolecta de chapulines (saltamontes) mediante uso de canastos.

Fig. 1. Collection of grasshoppers with basket.

en la diversión sobre todo escarabajos, grillos, y chapulines el $14 \%$, ninguno de ellos se refirió a algún aspecto místico o como amuletos.

El cultivo de estos insectos es muy escaso, sólo pudimos observar el de abejas, por lo que a esta pregunta sólo el $7 \%$ respondió positivamente.

En general, las respuestas a las preguntas del cuestionario que efectuamos en diferentes tiempos a las mismas personas, fueron muy semejantes.

Biodiversidad: los insectos empleados en la alimentación en el área de Zongolica en las localidades de estudio, fueron 57 (Cuadro 2), ellos corresponden a siete órdenes de la Clase Insecta. Los más utilizados son el orden Orthoptera que tuvo el mayor número registrado con 21 especies, luego el Hymenoptera con 13 especies, posteriormente el Lepidoptera con 10 especies, y finalmente el Coleóptera con ocho especies.

En algunos órdenes, tenemos que la biodiversidad es triespecífica como en el género Stilpnochlora del orden Orthoptera y biespecífica en los géneros Conocephalus, Melanoplus, Taeniopoda y Periplaneta del mismo orden, así como el género Umbonia del orden Homoptera, el género Macrodactylus del orden Coleoptera y los géneros Atta, Polistes y Polybia en el orden Hymenoptera. Treinta y seis géneros de los insectos comestibles de los diversos órdenes son uniespecíficos, o sea el $63.16 \%$

Consumo: la antropoentomofagia abarca las cuatro estaciones del año. Variando el tipo de especies que se ingiere en cada una de ellas. Los insectos son explotados en su época de mayor abundancia, que es cuando la gente hace menos esfuerzo en recolectarlos, ello da lugar a una calendarización de este hábito, modificándose los tiempos de ingestión, según los diferentes ciclos de vida y el estado aprovechable y buscado por la gente en cada especie, ya que como se ve en el Cuadro 2 se consumen en diferentes estados de desarrollo.

La ingestión de la mayoría de las especies es asada, pero otras son hervidas en agua con sal y en ocasiones fritos en manteca. Su sabor varía y puede que en el caso de los Orthoptera, tome el sabor del aderezo que se les ponga (salsas de diversos tipos de chile o hierbas aromáticas). En otros casos, saben a cacahuate, pepita de calabaza, pollo o chicharrón de puerco, y en el caso de los insectos sociales su sabor es semejante a la nuez, piñón o almendras (Ramos-Elorduy 1998). 
CUADRO 2

Biodiversidad de insectos comestibles registrados en la región de Zongolica, Veracruz, México

TABLE 2

Biodiversity of edible insects recorded in the Zongolica region, Veracruz, México

Orden-Familia Género Especie $\quad \begin{gathered}\text { Estado de Nombre Común } \\ \text { consumo }\end{gathered}$

\section{ORTHOPTERA}

\begin{tabular}{|c|c|c|c|c|c|}
\hline Tettigoniidae+ $\mathbf{\square} *$ & Stilpnochlora & azteca (Sauss.) & $\mathrm{n}, \mathrm{a}$ & Esperanza & Zongolica \\
\hline * & Stilpnochlora & thoracica (Serv.) & $\mathrm{n}, \mathrm{a}$ & Esperanza & Zongolica \\
\hline$\diamond$ & Stilpnochlora & quadrata (Scud.) & $\mathrm{n}, \mathrm{a}$ & Grillo verde & Zongolica \\
\hline 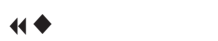 & Conocephalus & ictus (Scud.) & $\mathrm{n}, \mathrm{a}$ & Grillo & La Laguna, Ixtapaluka \\
\hline$\psi \diamond$ & Conocephalus & cinereus Thun. & $\mathrm{n}, \mathrm{a}$ & Grillo & Ixcohuapa \\
\hline$\Downarrow \diamond$ & Neoconocephalus & triops Linn. & $\mathrm{n}, \mathrm{a}$ & Esperanza & La Quinta \\
\hline$\psi \diamond$ & Scudderia & mexicana (Sauss.) & $\mathrm{n}, \mathrm{a}$ & Esperanza & Ixcohuapa \\
\hline$\Downarrow \diamond$ & Microcentrum & totonacum (Sauss.) & $\mathrm{n}, \mathrm{a}$ & Esperanza & Zongolica \\
\hline \multirow[t]{2}{*}{ Acrididae } & Sphenarium & sp. & $\mathrm{n}, \mathrm{a}$ & Chapulín de milpa & Amatitla \\
\hline & Melanoplus & femurrubrum De Geer & $\mathrm{n}, \mathrm{a}$ & Grillo de pata roja & La Quinta \\
\hline$+*$ & Melanoplus & sp. & $\mathrm{n}, \mathrm{a}$ & Chapulín saltón & Amatitla \\
\hline 4 & Aidemona & azteca (Sauss.) & $\mathrm{a}$ & Langosta & Zongolica \\
\hline+ & Osmilia (Abracris) & flavolineata De Geer & a & Grillo & Zongolica \\
\hline$\diamond$ & Taeniopoda & bicristata Burm. & a & Langosta & Ixcohuapa \\
\hline$+\square *$ & Taeniopoda & auricornis (Walk) & a & Grillo prieto & Mongolia \\
\hline $4+$ & Chromacris & colorata (Serv) & $\mathrm{a}$ & Chapulín rojo & Ixcohuapa \\
\hline \multirow[t]{2}{*}{ Blattidae } & Periplaneta & australasiae (Fab) & $\mathrm{a}$ & Cucarachita & $\begin{array}{l}\text { Tequilita, Ixcohuapa, } \\
\text { La Laguna, Ixtapaluka. }\end{array}$ \\
\hline & Periplaneta & americana Linn & $\mathrm{a}$ & Cucaracha & Ixcohuapa \\
\hline$\| \diamond$ & Blaberus & craniifer Burm. & a & Cucarachón & Ixtapaluka \\
\hline Phasmidae $\mathbf{4}$ & Bacteria & sp. & $\mathrm{a}$ & Palo tieso & Zongolica \\
\hline Gryllidae & Gryllus & assimilis (Fab.) & $\mathrm{a}$ & Grillo cantor & Zongolica \\
\hline \multicolumn{6}{|l|}{ HEMIPTERA } \\
\hline \multicolumn{6}{|l|}{ HOMOPTERA } \\
\hline \multirow[t]{2}{*}{ Membracidae } & Umbonia & reclinata Germ. & $\mathrm{a}$ & Toritos & Ixcohuapa \\
\hline & Umbonia & orizabae Buckton & a & Toritos & Zongolica \\
\hline Cicadidae & Fidicinoides & picea Walker & $\mathrm{n}, \mathrm{a}$ & Chicharra & Ixcohuapa \\
\hline \multicolumn{6}{|l|}{ MEGALOPTERA } \\
\hline Corydalidae & Corydalus & sp. & 1 & Manfe & La Laguna \\
\hline
\end{tabular}


CUADRO 2 (continuación...)

Biodiversidad de insectos comestibles registrados en la región de Zongolica, Veracruz, México

TABLE 2 (continued ...)

Biodiversity of edible insects recorded in the Zongolica region, Veracruz, México

Orden-Familia

Género

Especie

Estado de

Nombre Común

Localidad consumo

\section{COLEOPTERA}

Melolonthidae $\downarrow$

Isonychus

Phyllophaga

Goliathus

Macrodactylus

Macrodactylus

Geotrupes

Buprestidae

Cerambycidae

LEPIDOPTERA
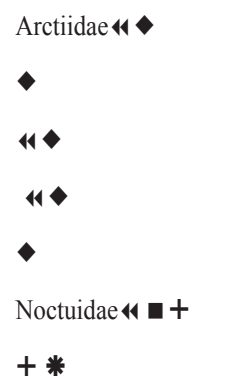

Hepialidae

Saturniidae*

Sphingidae+

HYMENOPTERA

\section{Elysius}

Amastus

Pelochyta

Estigmene

Pseudodirphia

Latebraria

Helicoverpa

Phasus

Arsenura

Manduca
Noctuidae $\mathbf{4}+$

Formicidae

घ+

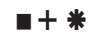

Vespidae+*

$+$

घ+

4

4

Atta

Polistes

Epipona

Vespula ocellatus Burm.

sp af lenis (Horn)

goliathus Zucht.

dimidiatus Gué.

lineatus Chev.

sp.

sp.

sp.

superba (Druce)

ochraceator Walk.

cervina (Edwards)

acraea Drury

mexicana Bouvier

amphipyroides Guenée

zea Boddie

triangularis Edwards

armida armida Cram

sexta Linneo

sp.

mexicana Smith

cephalotes Linneo

Polistes (Polisotius)

Polybia (Myrametra)

Polybia (Myrametra) major Beauv.

sp.

occidentalis nigratella Buysson

diguetana Buysson

sp.

sp.

$\begin{array}{cl}\text { a } & \text { Prietillos } \\ \text { 1, a } & \text { Chicharroncito } \\ \text { 1, a } & \text { Escarabajo Goliath } \\ \text { a } & \text { Chicalas } \\ \text { a } & \text { Chicalas } \\ \text { a } & \text { Boludo } \\ 1 & \text { Gusanos de los palos } \\ 1 & \text { Pochocho }\end{array}$

La Quinta Zongolica

Zongolica

La Quinta Zongolica

Zongolica

Zongolica

Zongolica

Ixtapaluka

Ixtapaluka

1 Gusano del palo mulato Zongolica

1 Gusanos de los palos Zongolica

a Gusanos de los palos Zongolica

a Oruga salina Zongolica

a Gusanos de los palos Zongolica

1 Cuetla

Ixtapaluka

1 Gusano elotero

Ixcohuapa

1 Gusanillo

Zongolica

1 Cuecla

Ixcohuapa

a

Gusano claro

Ixcohuapa

a rep Hormiga brava

Texhuacan

a rep Chicatanas

Ixcohuapa y Tequilita

a rep Chicatanas

Ixcohuapa

1, p, a Carniceras

Tequilita

1, p, a Carniceras

Amatitla

$\mathrm{a}, \mathrm{l}, \mathrm{p} \quad$ Avispita negra

Zongolica

a Avispita rayada

La Quinta

a, $1 \quad$ Panal blanci

Chilpa

Amatitla 
CUADRO 2 (continuación...)

Biodiversidad de insectos comestibles registrados en la región de Zongolica, Veracruz, México

TABLE 2 (continued ...)

Biodiversity of edible insects recorded in the Zongolica region, Veracruz, México

Orden-Familia

Género

Especie

Estado de

Nombre Común

Localidad

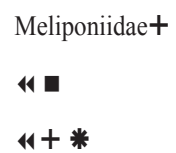

Apidae +

$\begin{array}{ll}\text { Partamona } & \text { bilineata (Say) } \\ \text { Melipona } & \mathrm{sp} . \\ \text { Trigona } & \mathrm{sp} . \\ \text { Apis } & \text { mellifera } \text { Linneo }\end{array}$

Zongolica

San Juan

Tequilita

La Laguna,

Ixtapaluka, Comalapa, Loma de Dolores,

Tepepamoxala y

Totolacatla

* Registradas para el Estado de Veracruz.

- Registradas para Bethel en la Selva Lacandona.

+ Registradas para el Estado de Chiapas.

- Nuevo registro para Insectos Comestibles de México 4 especies.

« Encontramos más especies de insectos comestibles de las que nosotros encuestamos, las cuales se agregaron en este cuadro.

$\mathrm{n}=$ ninfas, $\mathrm{a}=$ adultos, $\mathrm{a}$ rep $=$ adultos reproductores, $1=$ larvas, $\mathrm{p}=$ pupas.

Comercialización: en la mayoría de los casos, la gente sólo recolecta a los insectos comestibles para su uso familiar, sin embargo algunas especies, una vez preparadas y secas se comercializan en los tianguis y/o el mercado municipal de Zongolica, como son las "chicatanas", las "cueclas" y las "cuetlas" que se venden ocasionalmente, también los chapulines preparados, así como los "toritos". El precio varía según la cantidad y el tipo de cliente, e incluso de quien las vende (Fig. 2 y 3 ).

Protocultivos: comprobamos la existencia de protocultivos, que consisten en tener cuidados particulares a la especie con objeto de mantener su reproducción a lo largo del tiempo (Motte-Florac y Ramos-Elorduy 2000), como en el caso de la cucaracha $P$. australasiae F. Algunos habitantes de diversas localidades, sobre todo de Tequila e Ixcohuapa mantienen a los individuos de éstas en costales pequeños de yute, en donde agregan algunas piedras, para que las cucarachas tengan un resguardo y para alimentarlas le adicionan algunos granos de maíz y hojarasca recién recolectada, la que les brinda algunos microartrópodos que viven en ella, proveyéndolas de proteína animal (Fig. 4). De esta forma, los campesinos las tienen aseguradas. Su monitoreo es fácil, observándolas de vez en cuando, ver su abundancia, y luego las separan en el momento adecuado, las asan y se las comen en los estados de desarrollo en que se encuentren, siempre dejando a algunos especímenes. También con ellas preparan té para curar el asma o la tos, entre otras enfermedades respiratorias.

Además observamos que en el jonote, árbol de la especie Heliocarpus appendiculatus Turcz., se encuentran los gusanos conocidos como "cueclas", que son larvas de las mariposas que pertenecen a la especie $A$. armida armida Cramer (Fig. 5) y "las cuetlas" $L$. amphipyroides Guenée (Fig. 6) que se localizan en los árboles de Guásimo (Guasimo ulmifolia Lam), Jonote (H. appendiculatus) y Pochote (Bombax ellipticum H.B.K.) (Martínez 1979). 


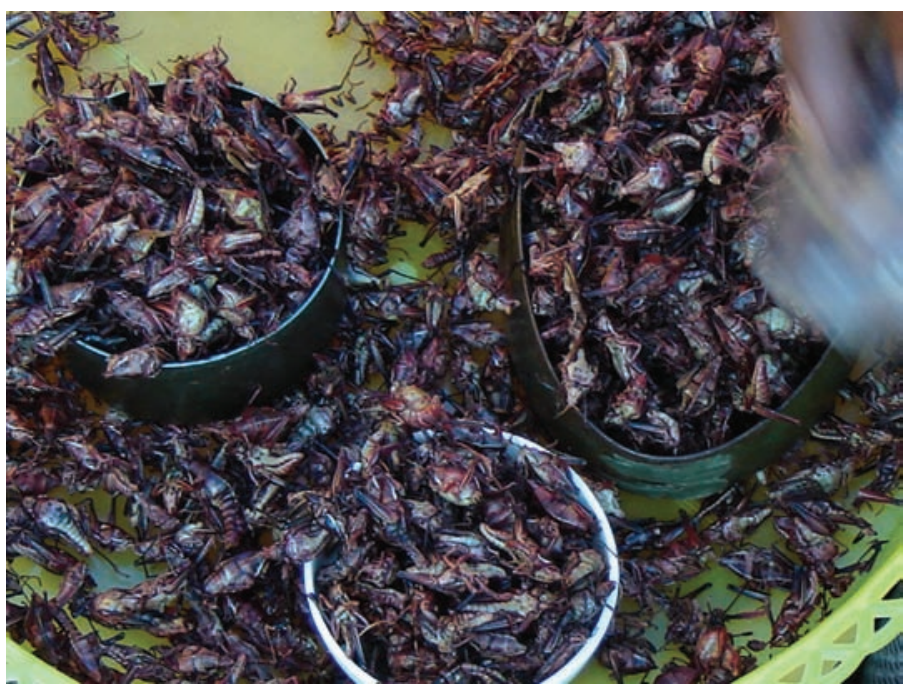

Fig. 2. Comercialización de chapulines en el mercado de Zongolica.

Fig. 2. Grasshoppers offered at the Zongolica market.

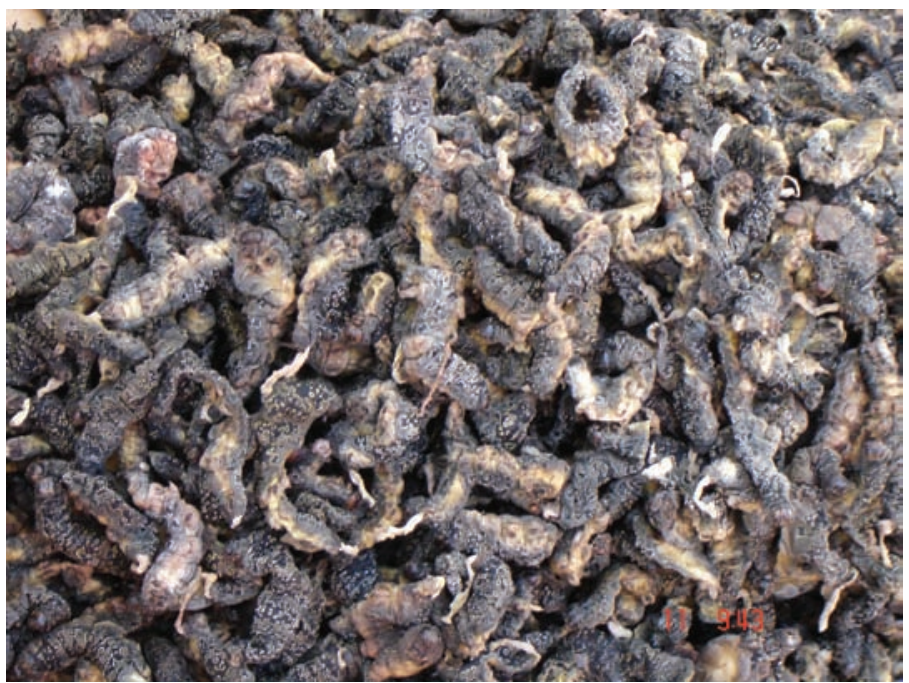

Fig. 3. Comercialización de "cuecla" en el mercado de Zongolica.

Fig. 3. Roasted caterpillars offered at the Zongolica market.

Ambas larvas presentan el hábito de ser gregarias, reuniéndose a una altura media del tronco, y colocándose alrededor de éste. Las larvas de la "cuecla" son de color muy oscuro, casi negras con manchas semiredondas de color gris y a su adulto se le denomina "mariposa del muerto". La “cuetla" regularmente está moviendo los osmeteria, por ello se le conoce como la "serpiente de las mil cabezas", sus larvas son muy coloridas y gruesas, de color verde con franjas en cada segmento de color negro y naranja. Ambas especies permanecen durante 


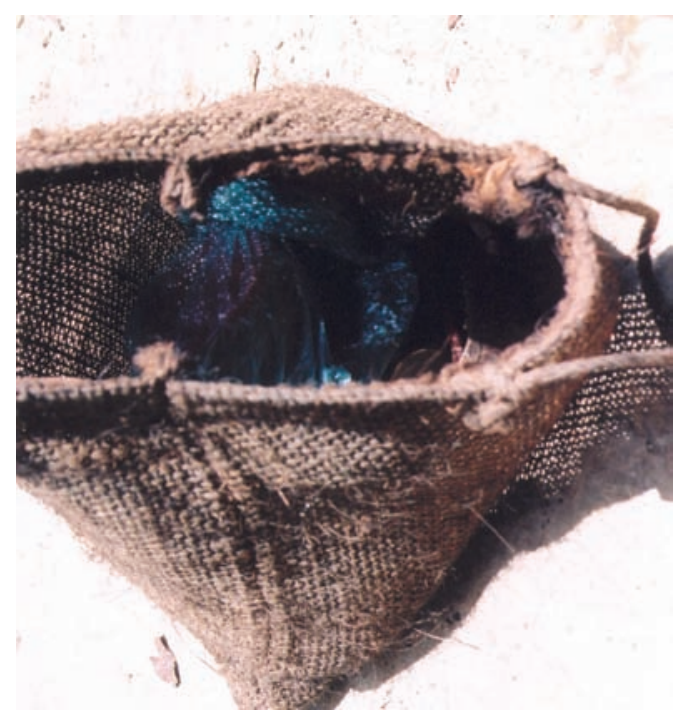

Fig. 4. Protocultivo de $P$. australasiae en Tequila e Ixcohuapa del municipio de Zongolica.

Fig. 4. Protoculture of $P$. australasiae on Tequila and Ixcohuapa, Zongolica.

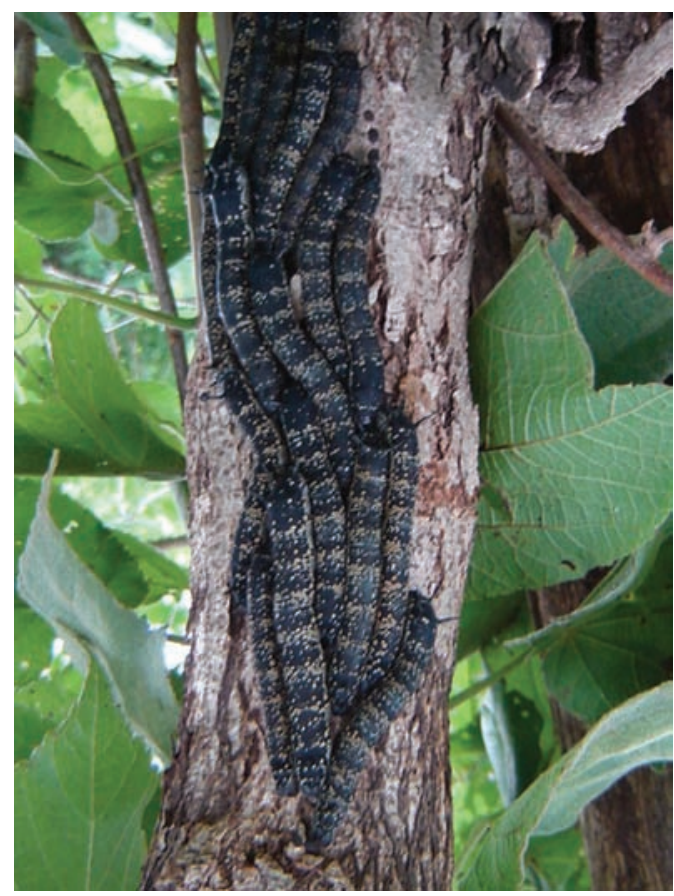

Fig. 5. Protocultivo de Arsenura armida armida en Heliocarpus appendiculatus Turcz.

Fig. 5. Caterpillar protoculture of Arsenura armida armida on Heliocarpus appendiculatus Turcz.

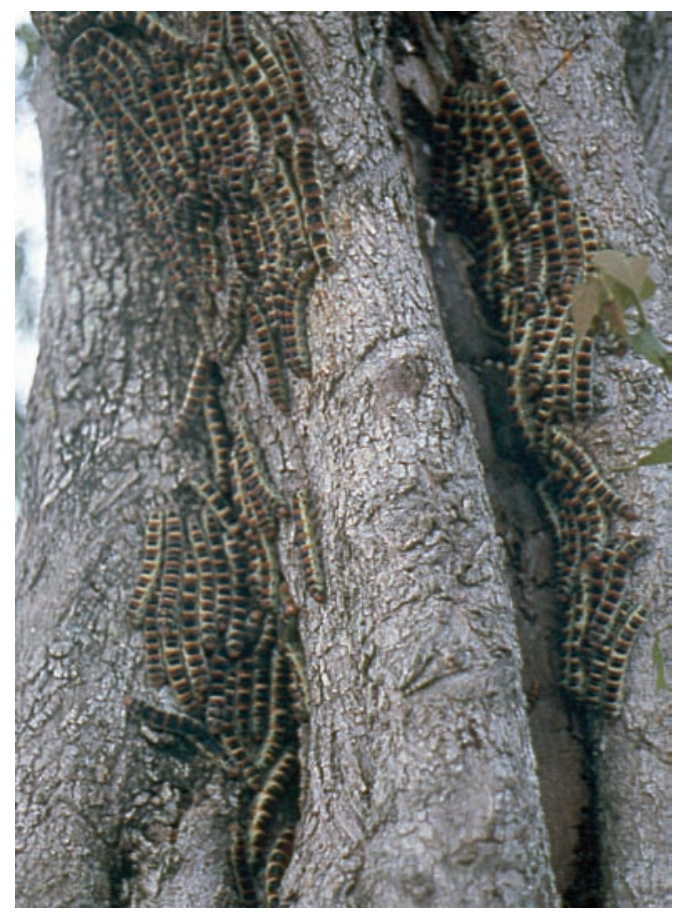

Fig. 6. Protocultivo de Latebraria amphipyroides en Guasimo ulmifolia Lam.

Fig. 6. Caterpillar protoculture of Latebraria amphipyroides on Guasimo ulmifolia Lam.

la mayor parte de día en los troncos, y en el mismo árbol, por lo cual es fácil monitorearlas y curiosamente no son atacadas ni ingeridas por los pájaros, lagartijas o camaleones, en donde quizás sea su gran tamaño el que les ayude, o la presencia de alguna sustancia tóxica, la cual se neutraliza en su preparación para ser ingeridos por los campesinos. Igualmente, ambas especies, durante la noche se mueven hacia las hojas más tiernas del árbol para alimentarse y luego en la madrugada regresan al tronco. Estas larvas son cuidadas por los campesinos y conservadas en su hospedero desde abril hasta mediados de junio, para que se desarrollen bien y posteriormente ya que están grandes la gente las recolecta, esto es a finales de junio y/o principios de julio, pero siempre dejan algunas larvas en dicho árbol, de manera que puedan llegar al estado adulto y reproducirse. 


\section{DISCUSIÓN}

Insectos Comestibles registrados: en esta investigación comprobamos la persistencia de la antropoentomofagia en esta área del Estado Veracruz, la cual debido a su localización geográfica se encontraba muy aislada, viendo que sus habitantes conservan aún sus tradiciones y hábitos alimenticios.

En los Insectos Comestibles de Zongolica encontramos 24 nuevos registros de Insectos Comestibles para México, o sea, el $44.44 \%$ del total de las especies registradas en esta región, ellos son los chapulines Stilpnochlora quadrata (Scudder), Conocephalus ictus (Scudder), Conocephalus cinereus Thunberg, Neoconocephalus triops Linneo, Scudderia mexicana (Saussere), Microcentrum totonacum (Saussere), Taeniopoda bicristata Burmeister, de las cucarachas $P$. australasiae y Blaberus craniifer Burmeister, la ingestión del fásmido Bacteria sp., del Orden Orthoptera, de la chinche Pyrrocoridae Dysdircus sp., de la cigarra Fidicinoides picea Walker, así como de las larvas y adultos de las mariposas de la familia Arctiidae que son Elysius superba (Druce), Amastus ochraceator Walker, Pelochytia cervina (Edwards), Estigmene acraea Drury y Pseudodirphia mexicana (Bouvier). Los escarabajos de los géneros Isonychus, Phyllophaga, Goliathus, Macrodactylus y Geotrupes, y la avispa del género Epipona.

Comparación del consumo de insectos: si vemos el número de especies rastreadas en la región de Zongolica 57 especies (47.11\%), de las 121 reportadas anteriormente para el estado de Veracruz (Ramos-Elorduy and Pino 1993) y lo comparamos con las encontradas en otra área trópico húmeda, como es un área de la Lacandonia (Ramos-Elorduy y Pino 2001b), vemos que es semejante 53 especies ( $29.78 \%$ ), de las 178 especies rastreadas para el estado de Chiapas (Ramos-Elorduy and Pino 2002b).

Pudimos ver que 12 de las especies rastreadas en Zongolica, ya habían sido reportadas con anterioridad para Veracruz (Ramos-Elorduy y Pino 1993) ellas son: Stilpnochlora azteca
(Saussure), Stilpnochlora thoracica (Serville), Melanoplus sp., Taeniopoda auricornis (Walker), F. picea, Helicoverpa zea Boddie, A. armida armida, Eciton sp., Atta cephalotes Linneo, Polistes (Polisotius) major Beauvier, Polybia (Mirametra) occidentalis nigratella (Buysson) y Trigona sp. Con la localidad de Bethel en la Selva Lacandona, Zongolica comparte nueve especies, ellas son: S. azteca, T. auricornis, L. amphipyroides, Eciton sp., Atta mexicana Smith, A. cephalotes, P. (M.) occidentalis nigratella, Melipona sp. y Apis mellifera (Linneo), y con el Estado de Chiapas comparte 18 especies más de este Estado (39.89\%), que son: S. azteca, S. quadrata, N. triops, Melanoplus sp., Osmilia flavolineata De Geer, T. auricornis, L. amphipyroides, H. zea, Manduca sexta Linneo, Partamona bilineata (Say), Eciton sp., A. mexicana, A. cephalotes, P. (P.) major, Polistes sp., P. (M.) occidentalis nigratella, Trigona sp., A. mellifera, ratificándose así la explotación y el uso de este recurso natural renovable de manera semejante por la gente de diferentes áreas rurales al compartir hábitos alimenticios similares y de acuerdo con la distribución geográfica de cada especie.

Es curioso observar que en ninguna de las dos localidades comparadas (Zongolica y Bethel) se cultiven a las abejas sin aguijón que constituyen un hábito milenario y que se cultivan en otras partes de este y otros Estados (Medina 1995).

Si se analizan los datos entre las localidades muestreadas, podemos ver que las especies de insectos comestibles, se concentran más en la capital del municipio, es decir en Zongolica, en donde censamos 25 especies registradas, le sigue la localidad de Ixcohuapa con 15 especies e Ixtapalupa con 11 especies. Sucesivamente en orden decreciente continua La Quinta con seis especies. Amatitla y Tequila con cuatro especies. Además existen otras cuatro localidades no mencionadas en donde se registró también el consumo de $A$. mellifera y una localidad donde se consume Melipona sp. (Cuadro 2) .

Protocultivos: en el caso de los tres protocultivos (P. australasiae, L. amphipyroides y $A$. 
armida armida), observamos que ello implica un conocimiento empírico, una observación acuciosa y un cuidado constante, así como una valoración alta del recurso.

Los insectos comestibles forman parte de una tradición alimenticia de los núcleos rurales de México y del mundo, el que se ha continuado de generación en generación. En el caso del protocultivo de la "cuetla" por ejemplo, los abuelos y las abuelas son los que conocen más y transmiten la información a las nuevas generaciones tanto en formas de consumo como en su conservación, pero en general podemos decir que carecen de cultivos de insectos comestibles, ello puede ser debido a que en realidad no les falta este recurso a lo largo del tiempo.

Mercadeo: además notamos que la comercialización de las especies, tanto a nivel personal, como a nivel de tianguis y mercados es baja y que en ningún caso observamos que alguna de las especies se almacenara una vez preservada, como sucede en otros sitios de México y del mundo (Menzel y D’Aluisio 1998, Ramos-Elorduy 2004, 2005).

\section{AGRADECIMIENTOS}

Agradecemos al convenio entre el Instituto de Biologia, de la Universidad Nacional Autónoma de México (UNAM) y la Universidad Veracruzana en Córdoba realizado a través de la Coordinación de la Investigación Científica de la UNAM, México el haber subsidiado este trabajo.

\section{RESUMEN}

Durante dos años y medio (2003-2005) se rastrearon a los insectos que se ingieren en el área de Zongolica, Veracruz: para ello se entrevistó a la gente de ese municipio (200), para conocer cuáles especies ingerían. El total de especies registradas, ascendió a 57 distribuidas en los órdenes Orthoptera, Hemiptera, Homoptera, Megaloptera, Coleoptera, Lepidoptera e Hymenoptera, correspondiendo el mayor consumo al primer orden. De las especies registradas,
24 son nuevos registros de insectos comestibles para México. Estos se consumen tanto en estado inmaduro como en estado adulto y generalmente se ingieren asados. Hay una secuenciación estacional en el consumo de las diferentes especies y algunas de ellas (las cucarachas, la cuetla, la cuecla, los chapulines, los toritos, etc.) se comercializan en los tianguis (puestos de venta callejeros informales que se realizan un día a la semana en diversos lugares del país) o en el día de mercado en Zongolica. Existe el "protococultivo" de tres especies: una cucaracha (Periplaneta australasiae Fabricius) y dos mariposas (Latebraria amphipyroides Guenée y Arsenura armida armida Cramer). La antropoentomofagía es un hábito ancestral en Zongolica.

Palabras clave: insectos comestibles, biodiversidad, Zongolica, Veracruz, México.

\section{REFERENCIAS}

Bahuchet, S. 1972. Etude écologique d'un campement de Pygmées Babinga (région de la Lobaye. République Centrafricaine). J. Agric. Trop. Bot. Appliq. 19: 509-559.

Bahuchet, S. 1978. Introduction à la ethnoécologie des pygmées Aka de la Lobaye Empire Centre Africain. Thèse Doctorat, Ecole Sup. d’ Hautes Etudes, Paris, Francia.

Costa-Neto, E. 2002. Manual de etnoentomología. Sociedad Entomológica Aragonesa, Zaragoza, España.

Gabdin, C. 1973. L' Ethnoentomologie. OPIE Cahiers de Liaison No.7: 15-17.

García, E. 1964. Modificación al sistema de clasificación climática de Köppen. UNAM, México D.F., México.

Gómez, P., R. Halut \& A. Colin. 1961. Production des protéines animales au Congo. Bull. Agric. Congo 52: 689-815.

Gordon, D. 1998. The eat -a -bug cookbook. Ten Speed, Berkeley, California, EEUU.

Hogue, Ch. 1987. Cultural Entomology. Ann. Rev. Entomol. 32: 181-199.

Kitsa, K. 1989. Contribution des insectes comestibles à l'amélioration de la ration alimentaire au KasaiOccidentale à Zaire. Afrique 239: 511-519.

Martínez, M. 1979. Catálogo de nombres vulgares y científicos de plantas mexicanas. Fondo de Cultura Económica, México D.F., México. 
Medina, M. 1995. Explotación de abejas sin aguijón: Scaptotrigona mexicana Guérin (ApidaeMeliponinae), en cajas racionales en la Región de Cuetzalán, México. Tésis de Maestría, Fac. de Ciencias, UNAM, México D.F., México.

Menzel, P. \& F. D’Aluisio. 1998. Man eating insects: the art and science of eating insects. Ten Speed, Berkeley California, EEUU.

Motte-Florac, E. \& J. Ramos-Elorduy. 2000. Is the traditional knowledge important?, p. 207-224. In J. Stepp, F. Wyndham \& R. Zarger (eds.). Ethnobiology, Benefits Sharing and Biocultural Diversity, Georgia, EEUU.

Ramos-Elorduy, J., J. Pino, C. Márquez, F. Rincón, M. Alvarado, E. Escamilla \& H. Bourges. 1984. Protein content of some edible insects of Mexico. J. Ethnobiology 4: 61-72.

Ramos-Elorduy, J. 1991. Los insectos como fuente de proteínas en el futuro. Limusa, México D.F., México.

Ramos-Elorduy, J. \& J.M. Pino. 1993. Algunos insectos comestibles del Estado de Veracruz. Resúmenes de la Primera reunión de investigadores sobre fauna Veracruzana. Instituto de Ecología, Xalapa Ver. 26-28 de abril, Veracruz, México.

Ramos-Elorduy, J. \& M. Conconi. 1994. Edible insects of the world. List of species, place of consumption and ethnic groups eaten them, p. 311-325. In Fourth Int. Cong. Ethnobiol., Lucknow, India.

Ramos-Elorduy, J. \& J. Pino. 1998. Insectos comestibles del estado de México y determinación de su valor nutritivo. An. Inst. Biol. UNAM 69: 65-104.

Ramos-Elorduy, J. 1998. Creepy Crawly Cuisine: The Gourmet Guide to Edible Insects. Inner Tradition International, Rochester, Vermont, EEUU.

Ramos-Elorduy, J. 1999. El consumo de insectos como un hábito ancestral, p. 275-305. In M. Rodríguez-
Shadow \& B. Barba (eds.). Chalchihuite. Homenaje a Doris Heyden Instituto Nacional de Antropología e Historia, Colección Científica, México DF.

Ramos-Elorduy, J. 2000. La Etnoentomología actual en México en la alimentación humana, en la medicina tradicional y en el reciclaje y alimentación animal, p. 3-46. In S. Stanford, A. Morales, J. Padilla \& M. Ibarra. Memorias del XXXV Congreso Nacional de Entomología, Acapulco, Guerrero, México.

Ramos-Elorduy, J. \& J. Pino. 2001a. Insectos comestibles del Estado de Hidalgo. An. Inst. Biol. UNAM 72: 43-84.

Ramos-Elorduy, J. \& J. Pino. 2001b. El consumo de insectos entre los lacandones de la comunidad de Bethel y su valor nutritivo. Etnobiología 1: 24-43.

Ramos-Elorduy, J. \& J. Pino. 2002a. Los insectos comestibles entre los nahuas. Entomol. Mex. 1: 103-104.

Ramos-Elorduy, J. \& J. Pino. 2002b. Edible insects of Chiapas, México. Ecol. Food Nutr. 41: 271-299.

Ramos-Elorduy, J. 2004. La etnoentomología en la alimentación, la medicina y el reciclaje, p. 329-413. In J. Llorente, J. Morrone, O. Yánez \& I. Vargas (eds.). Biodiversidad Taxonomía y Biogeografía de Artrópodos de México. Hacia una síntesis de su conocimiento IV. CONABIO/UNAM, México D.F., México.

Ramos-Elorduy, J. 2005. Insects: A hopeful food source, p.263-291. In M. Paoletti(ed.). Ecological Implications of Minilivestock (Role of Rodents, Frogs, Snails, and Insects for Sustainable Development). Science, Enfield, New Hamspire, EEUU.

\section{REFERENCIA DE INTERNET}

INEGI. 2000. Los municipios del Estado de Veracruz, p. 35-37. Consultado 6 junio 2007, www.inegi.mx 